\title{
Astrometric microlensing with the RadioAstron space mission
}

\author{
A. F. Zakharov ${ }^{1,2,3,4}$ \\ ${ }^{1}$ National Astronomical Observatories of Chinese Academy of Sciences, 20A Datun Road, \\ Chaoyang District, Beijing, 100012, China; \\ ${ }^{2}$ Institute of Theoretical and Experimental Physics, B. Cheremushkinskaya, 25, 117259, \\ Moscow, Russia; \\ ${ }^{3}$ Center of Advanced Mathematics and Physics, National University of Sciences and \\ Technology, Rawalpindi, Pakistan; \\ ${ }^{4}$ Bogoliubov Laboratory for Theoretical Physics, Joint Institute for Nuclear Research, 141980 \\ Dubna, Russia
}

\begin{abstract}
According to a revised schedule of the Russian Space Agency, in October 2008 the $10 \mathrm{~m}$ space telescope RadioAstron will be launched in a high eccentric orbit around the Earth. Acting together with ground based radio telescopes, the VLBI interferometer with a ground-space arm will operate. The interferometer will have extraordinary angular resolution of a few microarcsecond ( $\mu a s)$ at the shortest wavelength $(1.35 \mathrm{~cm})$. Since typical angular scales for gravitational microlensing are at the $\mu a s$ level for cosmological locations of sources and microlenses, in principle there is a chance to resolve microimages and (or) at least, detect astrometrical shift of bright point like images. In particular, gravitationally lensed systems, such as B1600+434, where in radio band a signature of microlensing is found, look suitable for direct observations of microlensing, since microlensing with the RadioAstron interferometer may be detected in the future (considering its high angular resolution and a relatively high sensitivity and assuming a ground support by the advanced radio telescopes).
\end{abstract}

Keywords. gravitational lensing, techniques: high angular resolution, astrometry, instrumentation: high angular resolution, instrumentation: interferometers

The RadioAstron space telescope is the high priority space project among Russian scientific space projects. According to the formal schedule of the Russian Space Agency (ROSCOSMOS), the space radio telescope RadioAstron should be launched in 2008. $\dagger$ This space based 10-meter radio telescope will be used for space - ground VLBI measurements. For observations four wavelength bands will be used. The bands correspond to $\lambda=1.35 \mathrm{~cm}, \lambda=6.2 \mathrm{~cm}, \lambda=18 \mathrm{~cm}, \lambda=92 \mathrm{~cm}$. If the interferometer operates with the 130-m Radio Telescope (EVLA) $\ddagger$ as a ground telescope, the minimal correlated flux can be at the levels of about 10, 1.3, 1.4, 3.2 mJy (RMS) at $0.327 \mathrm{GHz}$ (P-band), $1.665 \mathrm{GHz}$ (L-band), 4.830 GHz (C-band) 18.392-25.112 GHz (K-band), respectively, at the $8 \sigma$ level with an integration time about $300 \mathrm{~s}$. 9 Although we understand that, perhaps, the estimated sensitivity levels give an optimistic forecast (moreover, clearly the RadioAstron-ground interferometer will not operate at the maximum level of its sensitivity, since, obviously, the EVLA is not a dedicated facility for the RadioAstron space mission), we will use the estimates for our analysis as an initial point to demonstrate

$\dagger$ See the official web-site of the agency http://www.federalspace.ru/science0615E.asp, but now the mission was postponed (re-scheduled) to launch the spacecraft in October 2008, as it was indicated at the web-site of the RadioAstron project http://www.asc.rssi.ru/radioastron/. $\ddagger$ http://www.aoc.nrao.edu/evla/pbook.shtml

I http://www.asc.rssi.ru/radioastron/description/observations_eng.htm 
a potential possibility of discovering phenomenon such as splitting the images due to microlensing.

Assuming the concordant cosmological model parameters $\left(\Omega_{\mathrm{tot}}=1, \Omega_{\mathrm{matter}}=0.3, \Omega_{\Lambda}=\right.$ 0.7), a typical length scale for microlensing (the Einstein - Chwolson radius) is

$$
R_{E}=\sqrt{2 r_{g} \cdot \frac{D_{s} D_{l s}}{D_{l}}} \approx 3.4 \cdot 10^{16} \sqrt{\frac{M}{M_{\odot}}} h_{65}^{-0.5} \mathrm{~cm},
$$

where "typical" microlens and source redshifts are assumed to be $z_{l}=0.5, z_{s}=2$ (Treyer \& Wambsganss 2004), $r_{g}=\frac{2 G M}{c^{2}}$ is the Schwarzschild radius corresponding to microlens mass $M, h_{65}=H_{0} /((65 \mathrm{~km} / \mathrm{sec}) / \mathrm{Mpc})$ is the dimensionless Hubble constant, and the distance between two images is about $2 R_{E}$. We have a significant magnification $A$ (gravitational focusing), so that if the impact parameter $b$ is less than $R_{E}\left(b<R_{E}\right)$, then $A>3 \sqrt{5} / 5 \approx 1.34$.

The corresponding angular scale is

$$
\theta_{0}=\frac{R_{E}}{D_{s}} \approx 2.36 \cdot 10^{-6} \sqrt{\frac{M}{M_{\odot}}} h_{65}^{-0.5} \operatorname{arcsec} .
$$

If $M=10^{12} M_{\odot}$ (typical galactic masses), typical angular distances between images are about a few arcseconds, and it is called macrolensing. On the other hand, if $M=M_{\odot}$, typical angular distances between images are about microarcseconds, corresponding to the case of microlensing.

Using this length scale and a velocity scale such as an apparent velocity $\beta_{\text {app }}$, one could calculate the standard time scale corresponding to the time to cross the Einstein - Chwolson radius

$$
t_{E}=\left(1+z_{l}\right) \frac{R_{E}}{v_{\perp}}= \begin{cases}\approx 2 \sqrt{\frac{M}{M_{\odot}}} \beta_{\mathrm{app}}{ }^{-1} h_{65}^{-0.5} \text { weeks, } & \text { if } v_{\perp}=c \beta_{\mathrm{app}} \\ \approx 27 \sqrt{\frac{M}{M_{\odot}}} v_{600}{ }^{-1} h_{65}^{-0.5} \text { years, } & \text { if } v_{\perp} \sim 600 \mathrm{~km} / \mathrm{c} .\end{cases}
$$

Here we assume time scales are determined by an apparent velocity or a typical transverse velocity $\left(v_{600}=v_{\perp} /(600 \mathrm{~km} / \mathrm{c})\right)$, respectively. The time scale $t_{E}$ corresponds to the approximation of a point mass lens and a small size source in comparison with the Einstein - Chwolson radius. The approximation and the time scale estimate could be used if microlenses are distributed freely at cosmological distances and a fixed Einstein - Chwolson cone is actually located far enough from the other ones.

For gravitationally lensed systems, if microlenses form a significant part of a gravitational lens mass, a caustic network generated by microlenses and photometric microlensing (fluctuations in light curves corresponding to different images) arise if a source crosses these caustics. If we use the simple caustic microlens model (like the straight fold caustic model), there are two time scales, depending on the "caustic size" and source radius $R_{\text {source, }}$, respectively. Under the condition that the source radius is larger or about the same as the "caustic size" $r_{\text {caustic }}$ (if we use the following approximation for the magnification near the caustic $\mu=\sqrt{\frac{r_{\text {caustic }}}{y-y_{c}}}$ where $y>y_{c}$ and $y$ is the perpendicular direction to the fold caustic), if $R_{\text {source }} \gtrsim r_{\text {caustic }}$, then the relevant time scale is the "crossing caustic time" (Treyer \& Wambsganss 2004)

$$
t_{\text {cross }}=\left(1+z_{l}\right) \frac{R_{\text {source }}}{v_{\perp}\left(D_{s} / D_{l}\right)} \approx 0.62 R_{15} v_{600}^{-1} h_{65}^{-0.5} \text { years } \approx 226 R_{15} v_{600}^{-1} h_{65}^{-0.5} \text { days, }
$$


where $D_{l}$ and $D_{s}$ correspond to $z_{l}=0.5$ and $z_{s}=2$ respectively and $R_{15}=R_{\text {source }} / 10^{15} \mathrm{~cm}$.

However, if the source radius $R_{\text {source }}$ is much smaller than the "caustic size" $r_{\text {caustic }}$ ( $\left.R_{\text {source }} \ll r_{\text {caustic }}\right)$, one could use the "caustic time", namely the time when the source is located in the area near the caustic, and the time scale then corresponds to

$$
t_{\text {caustic }}=\left(1+z_{l}\right) \frac{r_{\text {caustic }}}{v_{\perp}\left(D_{s} / D_{l}\right)} \approx 0.62 r_{15} v_{600}^{-1} h_{65}^{-0.5} \text { years } \approx 226 r_{15} v_{600}^{-1} h_{65}^{-0.5} \text { days },
$$

where $r_{15}=r_{\text {caustic }} / 10^{15} \mathrm{~cm}$.

These time scales $t_{\text {cross }}$ and $t_{\text {caustic }}$ could be about days (or even hours) if $v_{\perp}$ is determined by an apparent motion of superluminal motion of knots in jet.

Thus, $t_{\text {cross }}$ could be used as a lower limit for typical time scales for the simple caustic microlens model, but there are two length parameters in the problem. In general we do not know their values, and so we could not evaluate $R_{\text {source }}$ only from the time scales of microlensing because time scales could correspond to two different length scales. However, if we take into account variation amplitudes of luminosity, one could say that, in general, $t_{\text {cross }}$ corresponds to smaller variation amplitudes than $t_{\text {caustic }}$. This is because if the source area is large there is a "smoothness" effect - since only a small fraction of source area is located in the high amplification region near the caustic.

In conclusion, first, one could point out that gravitational lensed systems are the most perspective objects to search for microlensing. Astrometric microlensing could be detected in the gravitational lens system such as B1600+434 in case proper motions of source, lens and an observer are generated mostly by a superluminal motion of knots in jet. But in this case, with the density flux estimates obtained by Koopmans \& de Bruyn (2000), one could say that based on the optimistic estimates of the RadioAstron interferometer acting with ground support of the EVLA (or similar ground based radio telescope), such motions in knots may be detectable.

Second, in case there is microlensing in the B1600+434 system, for example, then astrometric microlensing in the system could be about $\theta_{\text {shift }} \sim 20-40$ pas (Treyer \& Wambsganss 2004). In the best case the RadioAstron interferometer will have enough sensitivity to detect such an astrometric displacement as it was noted earlier, and moreover, as estimates showed, even splitting of the microimages could be detectable.

Third, in principle microlensing for distant sources could be the only tool used to evaluate $\Omega_{L}$ (including dark matter component) from event rate for microlensing. To solve this problem with the RadioAstron interferometer one should analyze variabilities of compact sources with a core size $\lesssim 40 \mu a s$ and with high enough flux densities. We should apply the most reliable model for variabilities of the sources such as scintillations, microlensing etc. A fraction of the sources in the list of extragalactic targets for VSOP and RadioAstron is suitable for this analysis. If the analysis would indicate that other explanations (such as scintillations) are preferable and future observations with RadioAstron interferometer would show that there are no features for astrometric microlensing, one could conclude that the Hawkins claim (supporting microlensing as a source of variabilities for a significant part of distant quasars) should be ruled out. But if an essential fraction of variability could be explained by microlensing, the sources could serve as first order candidates to search for astrometric microlensing. Therefore, one could say that astrometric microlensing (or direct image resolution with RadioAstron interferometer) is the crucial test to confirm (or rule out) the microlens hypothesis for gravitational lensed systems and for point like distant objects. Astrometric microlensing due to MACHO action in our Galaxy is not very important for observations with the space interferometer RadioAstron. This is because first, such probabilities are not high and secondly, typical time scales are longer than the estimated life time of the RadioAstron space mission. 
Thus, right after the RadioAstron launch, we should take the first opportunity to detect microlensing (splitting of the micro-images) in a direct way. So the main objective of this paper to pay attention to such a challenging possibility because, preflight time is very short now and perspective targets should be analyzed carefully in advance by observational and theoretical means. Since there are only few point like bright sources at cosmological distances and gravitational lensed systems with point like components demonstrating microlens signatures, these sources should be analyzed with great care way to search for candidates for which the microlens model is preferable in comparison with alternative explanations of variabilities.

We would like to point out also that there are some non-negligible chances of observing mirages (shadows) around the black hole at the Galactic Center and in nearby AGNs in the radio-band (or in mm-band) using RadioAstron (or Millimetron) facilities (see the detailed discussion by Zakharov et al. $(2005 \mathrm{a}, \mathrm{b})$ ). Since a shadow size should be about $50 \mu a s$ for the black hole in the Galactic Center, analyzing the shadow size and shape, one could enable us to evaluate a spin and a charge of the black hole.

Finally, we note that the next space Russian dish for VLBI (ground-space) facilities will be in action after about one decade, since the Russian Space Agency and the Russian Academy of Sciences have planned to launch the cryogenic telescope Millimetron for observations at mm (and sub-mm) wavelength bands in 2016. $\dagger$ This instrument will be placed near the Lagrange point $L_{2}$ of the Sun - Earth system. The Millimetron itself will have bolometrical sensitivity of about $5 \times 10^{-9} \mathrm{Jy}$ at $0.3 \mathrm{~mm}$ wavelength with an integration time of about 1 hour. The ALMA - Millimetron interferometer with the space-ground arm will have an angular resolution of $10^{-9}$ arcseconds with a sensitivity of about $0.1 \mathrm{mJy}$ level at $0.5 \mathrm{~mm}$ wavelength with an integration time of about $300 \mathrm{~s}$. This will give us an opportunity to analyze not only microlensing but also nanolensing due to actions of gravitating bodies with planet masses.

\section{Acknowledgments}

The author is grateful to the National Natural Science Foundation of China (NNSFC) (Grant \# 10233050) and the National Key Basic Research Foundation (Grant \# TG 2000078404) for a partial financial support of the work and profs. J.-X. Wang and J. Zhang for fruitful discussions.

\section{References}

Koopmans, L. V. E., \& de Bruyn, A. G., 2000, A\& $A, 358,793$

Popović L. C. Jovanović, P., Mediavilla, E., et al. ApJ, 2006, 637, 620

Treyer, M. \& Wambsganss, J., 2004, A\& $A, 416,19$

Zakharov, A. F., 1995, $A$ \& $A, 293,1$

Zakharov, A. F., 1997a, Ap \&S SS, 252, 369

Zakharov, A. F., 1997b, Gravitational Lensing and Microlensing, Janus-K, Moscow

Zakharov, A. F., 2003, Publ. Astron. Obs. Belgrade 75, 27; astro-ph/0212009

Zakharov, A. F., 2005, in A.I. Studenikin (ed.), Proc. of the Eleven Lomonosov Conference on Elementary Particle Physics, (World Scientific, Singapore) p. 106; astro-ph/0403619

Zakharov, A. F., 2006a, Astron. Reports, 50, 79

Zakharov, A. F., 2006b, Physics of Particles and Nuclei Lett., (in press); astro-ph/0610857

Zakharov, A. F., Nucita, A. A., De Paolis, F., \& Ingrosso, G., 2005a, New Astron., 10, 479

Zakharov, A. F., Nucita, A. A., De Paolis, F., \& Ingrosso, G., 2005b, A $\mathcal{E}$ A, 442, 795

Zakharov, A. F., Popović, L. C.., \& Jovanović, P., 2004, A \& $A$, 420, 881

Zakharov, A. F. \& Sazhin, M. V., 1998, Physics-Uspekhi, 41, 945

$\dagger$ http://www.federalspace.ru/science0615E.asp. 